\title{
Enhancing teamwork to create a high-performance team in the acute stroke unit at Worcestershire Royal Hospital - quality improvement project
}

\author{
Author: Soorya M Sureshkumar ${ }^{\mathrm{A}}$
}

\section{Introduction}

The acute stroke unit (ASU) in Worcestershire Royal Hospital has a multidisciplinary team including consultants, junior doctors, nurses, physiotherapists, occupational therapists, and speech and language therapists focusing on delivering hyperacute stroke treatment and rehabilitation. However, it is felt there are difficulties in multiple levels such as staffing issues, disorganised ward rounds and teamwork, lack of communication between teams, lack of local induction, hindering our multidisciplinary team to become a high-functioning team. This quality improvement project (QIP) focused on identifying the areas of improvement and to try and devise strategies to make highperformance team utilising resources within.

\section{Methods}

Plan, study, do, act (PDSA) cycle 1 focused on identifying the areas of improvement through a survey among members of the multidisciplinary team. Likert-scale questionnaire divided and organised into four different logical constructs, general organisation, junior doctor accessibility, patient care plans were used for the survey. Resurvey with the same questionnaire followed the implementation of changes to identify the outcome of changes which led to improving morning huddle.

PDSA cycle 2 improved the morning huddle with introducing a local checklist from SAFER patient flow bundle, NHS improvement and Royal College of Physicians recommendations for best practice in medicine. ${ }^{1,2}$ Data over the QIP period for patient safety elements such as venous thromboembolism documentation and completion of discharge summaries, and patient experience element on number of compliments received, were collected.

\section{Outcome}

The PDSA cycle 1 showed significant change within the four variables: general organisation $(t(13)=4.266 ; p<0.05)$; junior doctor accessibility $(\mathrm{t}(13)=7.659 ; \mathrm{p}<0.05)$; efficiency $(\mathrm{t}(13)=$ 2.474; $\mathrm{p}<0.05)$; and patient care $(\mathrm{t}(13)=10.617 ; \mathrm{p}<0.05)$.

There was a $16 \%$ increase in the venous thromboembolism prevention documentation and a $1.3 \%$ increase in discharge summary completion in 24 hours. Additionally, there were 27 more compliments received for the ASU during the QIP period.

\section{Conclusion and discussion}

There is unequivocal evidence of improved outcomes when patients are treated in a stroke unit by multidisciplinary teams. ${ }^{3}$ Evidence from observational studies suggests that explicit communication (eg task allocation, prioritisation of patients and task ownership) facilitates behaviours associated with patient safety (eg prescription of thromboprophylaxis, removal of unused cannulae etc). ${ }^{2}$ The significant change in four variables - organisation, patient care, efficacy and junior doctor accessibility - by cycle along with the introduction of improvement strategies in morning huddle showed $16 \%$ increase in the venous thromboembolism prevention documentation and a $1.3 \%$ increase in discharge summary completion. Additionally, there were 27 more compliments received for the ASU during the QIP period. As a part of cycle 2, a local induction booklet was introduced.

\section{Conflicts of interest}

None declared.

\section{References}

1 NHS Improvement. SAFER patient flow bundle. NHS. https:// improvement.nhs.uk/resources/safer-patient-flow-bundleimplement [Accessed 15 September 2019].

2 Royal College of Physicians, Royal College of Nursing. Ward rounds in medicine: Principles for best practice. London: RCP, 2012.

3 Clarke DJ. The role of multidisciplinary team care in stroke rehabilitation. Progress in Neurology and Psychiatry 2013;17:5-8. 